\title{
El principio de Mach en la
Relatividad General y en la Mecánica Relacional
}

\author{
Ángel José Chacón Velasco \\ Universidad Pedagógica y Tecnológica de Colombia
}

Resumen: Desarrollamos algunos aspectos que vinculan la nueva mecánica (1998) formulada por André Koch Torres Assis, con respecto a las mecánicas de Newton y Einstein, y que se presenta como una mecánica relacional en el espíritu de Leibniz y Berkeley respecto de la naturaleza del espacio, el tiempo y el movimiento.

Analizamos la emergencia del principio de Mach desde esta teoría, expresado matemáticamente a través de la ley de Weber para la gravitación.

Además hacemos un análisis crítico de algunas implicaciones físicas, epistemológicas y pedagógicas de esta teoría con respecto a las evidencias experimentales, la cosmología y la enseñanza de la mecánica.

Palabras clave: Principio de Mach, Mecánica relacional, Relatividad General, epistemología de la física.

Abastract: This article develops a series of aspects that link the New Mechanics formulated by André Koch Torres Assis (1998) to the Newton and Einstein's Mechanics, presented as a Relational Mechanics in the spirit of Leibniz and Berkeley in what refers to the nature of space, time and movement. We analyze the emergence of Mach's Principle from this theory, mathematically expressed by Weber's Law of Gravitation.

We also make a critical analysis of some of the logical, epistemological and pedagogical implications of this theory with respect to experimental evidence, cosmology and the teaching of Mechanics.

Key words: Mach’s Principle, Relational Mechanics, General relativity, epistemology of Physics 


\section{Introducción}

L

a importancia de la crítica que hace Ernst Mach de la mecánica de Newton consiste en que, en esta, el espacio absoluto de Newton es abolido, en la formulación de la

Anercia, sin embargo dentro de este análisis encontramos un principio fundamental que ha sido el hilo director de importantes desarrollos teóricos en torno a la mecánica celeste y más recientemente a la cosmología, el principio de Mach, muchas veces citado pero sobre el que se ha tejido una aureola de incomprensión. Uno de los propósitos fundamentales de este trabajo consiste en aclarar este principio en el contexto de la mecánica relativista, de la teoría general de la relatividad y en la nueva mecánica relacional.

Las ideas de Mach acerca de esta cuestión se encuentran básicamente en su libro Desarrollo histórico-crítico de la mecánica [4], sin embargo en ninguno de sus escritos Mach dejo claro lo que terminó siendo llamado el principio de Mach; a pesar de esto sus ideas contra las nociones del espacio y el tiempo absolutos de Newton, a favor de una física relacional, el reconocimiento de la realidad de las fuerzas ficticias o de inercia, la suposición de que la experiencia del balde de Newton muestra una conexión entre la curvatura del agua al girar el balde con la presencia de las entonces estrellas fijas, es lo que terminó siendo conocido en términos generales como principio de Mach.

Fue M Schlick en 1915 [9] quien primero utilizó esta expresión, refiriéndose aparentemente a la propuesta general de Mach para una relatividad de todos los tipos de movimiento, o sea que no existen movimientos absolutos en relación al espacio, y solo respecto de la materia en relación consigo misma.

De acuerdo con Schlick, una consecuencia de esta propuesta es que se debe "asumir que la causa de la inercia es resultado de la interacción entre las masas".

Luego esta expresión empezó a ser ampliamente utilizada desde 1918 por Einstein, quien fundamenta la teoría general de la relatividad en tres principios:

a) El principio de relatividad, que establece que las leyes de la naturaleza son solo afirmaciones sobre coincidencias espacio-temporales; por tanto las leyes encuentran solo su expresión natural en ecuaciones generalmente covariantes.

b) El principio de equivalencia: La inercia y el peso son idénticos por naturaleza, se sigue necesariamente de esto y del resultado de la teoría especial de la relatividad que es el "tensor fundamental" simétrico $g_{\mu \nu}$ determina las propiedades métricas del espacio, el comportamiento inercial de los cuerpos en este espacio, asi como los efectos gravitacionales, denotado este espacio por el tensor fundamental como el "campo -G"

c) Principio de Mach: el campo -G esta totalmente determinado por las masas de los cuerpos. Como la masa y la energía son idénticos de acuerdo con la relatividad especial y la energía es descrita formalmente a través del tensor de energía simétrico $T_{\mu \nu}$, esto significa que el campo - G esta condicionado y determinado por el tensor de energía de la materia.

Otras formas como se ha "formulado" por distintos autores el principio de Mach son: 
"Los referenciales inerciales son aquellos que no están acelerados en relación a las estrellas fijas, esto es, en relación a una media definida convenientemente de toda la materia del universo" [4].

"Si la inercia no se da por el movimiento en relación con el espacio absoluto, esta debe ser producida por la materia alrededor del cuerpo".

"El programa de Mach significa la intención de entender todos los efectos inerciales como siendo causados por la interacción gravitacional".

"Las propiedades inerciales de la materia en el ámbito local son derivadas de alguna forma a partir de la existencia de las masas distantes del universo y de sus distribuciones espaciales".

"El movimiento y por consiguiente la masa de cada uno de los cuerpos, esta determinada, causada, producida, por los otros cuerpos en el universo".

Y el mismo Mach en su análisis histórico crítico de la mecánica 1883 [4]:

"Nadie puede decir algo sobre el espacio absoluto o sobre el movimiento absoluto, que no sean meras abstracciones sin manifestación posible en la experiencia. Todos nuestros enunciados fundamentales de la mecánica, como hemos mostrado detalladamente, son experiencias sobre posiciones y movimientos relativos de los cuerpos".

\section{La mecánica de Newton y las criticas de Mach}

Es ampliamente conocida, con sus posteriores desarrollos, la mecánica clásica formulada por Newton en su libro Principios Matemáticos de Filosofía Natural (1687) [6], donde Newton define axiomáticamente las cantidades absolutas de fuerza centrípeta y de su medida, de cantidad acelerativa de una fuerza y de la cantidad motora de una fuerza centrípeta respectivamente.

Luego bajo la figura de un importante escolio hace las definiciones controversiales de espacio, tiempo y movimientos absolutos y relativos, para presentar finalmente sus tres axiomas o leyes de movimiento, que constituyen el núcleo principal de su teoría y que hoy por hoy se siguen considerando validas para un entorno clásico y en sistemas de referencia inerciales por el definidos. Este sistema teórico de la mecánica sigue siendo el paradigma que se enseña en distintos programas de ingeniería en cursos básicos y a través de sus desarrollos posteriores conocidos como mecánica analítica, lagrangiana y hamitoniana.

Después de las tres leyes Newton presenta algunos corolarios. El primero es la ley del paralelogramo de las fuerzas o de las aceleraciones y en el quinto introduce los referenciales inerciales, aunque Newton en los principia nos les de este nombre, "el movimiento de los cuerpos encerrados en un espacio dado son los mismos entre si, este, el espacio en reposo, o en movimiento uniforme en línea recta sin ningún tipo de movimiento circular”, o sea, es posible utilizar las leyes de Newton no solo en el espacio absoluto sino en cualquier sistema de referencia que se mueva uniformemente en relación al espacio absoluto.

Para poder aplicar sus leyes, Newton propone la ley de gravitación universal, como una fuerza proporcional al producto de las masas e inversamente proporcional al cuadrado 
de la distancia entre los cuerpos, en dirección de la línea recta que une los cuerpos a su vez considerados cuerpos simétricamente esféricos e incluso como puntos materiales.

De importantes implicaciones cosmológicas son los planteamientos donde contempla Newton la posibilidad de despreciar la influencia de las estrellas distantes sobre los cuerpos del sistema solar, debido a su distribución aleatoria en el cielo, lo cual implicaría su cancelación mutua.

En los Principia en el inicio del libro I y antes de los axiomas del movimiento, Newton presenta el experimento del balde (mas tarde se conocería como el experimento del balde de Newton) para darle un soporte empírico a los conceptos de espacio y tiempo absolutos entendidos por el desde sus inicios como problemáticos.

En esencia, este experimento consiste en un balde lleno de agua y suspendido por una cuerda larga y retorcida de tal modo que cuando se suelta el balde comienza a girar a gran velocidad, luego la superficie del agua, de plana comienza a seguir el movimiento del balde y se eleva por los bordes del mismo, tornándose en una superficie cóncava, mostrando así que está girando en el espacio, aun estando en reposo relativo al balde con el cual esta en contacto. Con este experimento Newton creyó haber probado, experimentalmente, que la rotación es un tipo de movimiento absoluto. A mediados del siglo XIX Foucault realizo su celebre experimento del péndulo, presentado a su vez como otra demostración de la rotación de la tierra respecto de algo.

Al respecto del experimento del balde y sus implicaciones para Newton como la posibilidad de distinguir entre una rotación absoluta y una relativa, fue Ernst Mach quien en su estudio critico de la mecánica, La ciencia de la mecánica de 1883, encontró que esa experiencia relatada por Newton no probaría que el agua se elevaría debido a las fuerzas que se engendran mientras gira en el espacio absoluto. Mach planteaba, por ejemplo, que la misma observación debería hacerse si el balde de agua estuviera quieto y las estrellas fijas estuvieran en rotación respecto del cubo.

Mach planteaba que de la experiencia del balde solamente se puede inferir que solo los movimientos relativos son los observados y que por tanto solo ellos deben figurar en las leyes físicas, "los principios de la mecánica pueden de hecho ser concebidos de tal manera que para rotaciones relativas surgen las fuerzas centrifugas" y que "al intentar fijar el balde y hacer girar el cielo de las estrellas fijas, se podrá entonces probar la ausencia de fuerzas centrifugas".

Entre otras cosas Mach nos dice

"La experiencia de Newton con el balde de agua que gira, nos enseña simplemente que la rotación relativa del agua respecto de las paredes del balde no despierta ninguna fuerza centrífuga efectiva, pero que esta es, en cambio, provocada por la rotación relativa respecto de la masa de la tierra y de los demás astros"[4].

En la mecánica newtoniana, si dejáramos el balde con agua parado con relación a la tierra y girásemos el conjunto de las estrellas distantes (en relación a la Tierra y al balde con agua) alrededor del eje del balde, nada debería acontecer en la superficie del agua de acuerdo con el teorema XXX de los Principia. Sin embargo para Mach el agua sí debería subir por las paredes del balde, así como en la experiencia original de Newton, siempre 
que la velocidad angular relativa entre el agua y las estrellas distantes sea la misma en los dos casos.

Finalmente, la idea de que la inercia de cualquier cuerpo (su masa inercial o su resistencia a sufrir aceleraciones) es debida a una interacción con el universo como un todo, pasó a ser conocida como principio de Mach. Sin embargo Mach en sus estudios, no logró plasmar este principio matemáticamente, para hacerlo necesitaba mostrar que al girar el conjunto de las estrellas alrededor de la Tierra, aparecería una fuerza centrífuga real que empujaría el agua hacia las paredes del balde y que achataría la Tierra en los polos, también tendría que demostrar que aparecería una fuerza de Coriolis real capaz de cambiar el plano de oscilación del péndulo de Foucault, ninguna de estas fuerzas aparece en la mecánica de Newton, y Mach, mas allá de hacerlo notar, no planteó ninguna alternativa, ni matemática de naturaleza heurística, pero sí sirvió de fuerte inspiración a otros científicas, entre estos a Einstein.

\section{La influencia de las ideas de Mach en la relatividad general}

Albert Einstein formuló la teoría de la relatividad general en 1916, con el objetivo, entre otros, de vincular cuantitativamente el principio de Mach.

En su libro La esencia de la relatividad, 1922, [5] plantea al respecto de las objeciones de Mach a la mecánica de Newton, que una teoría alternativa basada en esta crítica debería incluir las siguientes ideas:

1. La inercia de un cuerpo debe aumentar cuando se acumulan en su vecindad masas ponderables,

2. Un cuerpo debe estar sometido a la acción de una fuerza aceleradora cuando las masas vecinas se aceleran y, en realidad, dicha fuerza debe tener el mismo sentido que la aceleración,

3. Un cuerpo hueco animado de movimiento rotatorio debe producir en su interior un campo de coriolis, el cual desvía a los cuerpos en movimiento en el sentido de la rotación y deberá además producir un campo de fuerzas centrifugas radial.

Según Einstein, debería esperarse una cuarta consecuencia al vincular el método de razonar de Mach en la formulación de una teoría que incorpore su principio y esta sería:

4. Que un cuerpo en un universo vacío no tendría inercia, o toda la inercia de un cuerpo proviene de su interacción con otras masas del universo.

Einstein creyó hasta su muerte en 1955, que la primera de las ideas de Mach al respecto, se implementaba en la teoría general de la relatividad, aunque manifestó que su magnitud seria tan pequeña que no podría esperarse su confirmación mediante experiencias de laboratorio.

El significado de este primer punto lo podemos ilustrar mediante un ejemplo; dejemos caer un cuerpo en caída libre en el vacío, su aceleración será $a_{1}=9,8 \mathrm{~m} / \mathrm{s}^{2}$, si llevamos el cuerpo al interior de un cascarón vacío, y lo soltamos libremente, su aceleración de caída libre debe disminuir de acuerdo con Einstein, o sea $a_{1}>a_{2}$. De igual manera en la mecánica de Newton, la única fuerza actuando sobre la masa del cuerpo es el peso, (originado en la atracción gravitacional de la Tierra sobre el cuerpo), de la segunda ley de Newton $P=m a$, de tal forma que $a=P / m$. Si aumentamos la inercia del cuerpo (su masa inercial) al rodearlo 
con el cascarón entonces su aceleración de caída libre debería disminuir. Esta idea es intuitivamente plausible y está de acuerdo con el principio de Mach, es decir, si la inercia de un cuerpo proviene de su interacción con el universo distante, entonces se acumulamos cuerpos a su alrededor su inercia tiene que aumentar.

Sin embargo desde 1962, con el trabajo de Brans, se sabe que este efecto no se da ni en la relatividad general, como tampoco en la mecánica de Newton, ya que en estas teorías $a_{1}=a_{2}$.

La segunda consecuencia si se da en la relatividad general pero su interpretación no es única.

Respecto de la tercera consecuencia, Thirring en 1918 y más recientemente Mashhoon [8] demuestran que estos efectos si aparecen en la relatividad general, y que en la mecánica de Newton no aparece correspondencia a la fuerza axial en dirección de la velocidad angular, en sistemas de referencia no inerciales, por lo que este término es considerado espurio.

Por estas razones se concluye que la relatividad general no puede explicar la experiencia del balde de Newton en todos los referenciales.

La cuarta consecuencia tampoco se da en la teoría de Einstein, ya que él mismo demostró que de sus ecuaciones de campo se infiere que una partícula de prueba en un espacio vacío tiene propiedades inerciales. Además de Sitter, introduciendo la constante cosmológica encontró soluciones a las ecuaciones de campo modificadas en ausencia de materia. Kurt Gödel también halló soluciones a las ecuaciones de campo, interpretadas para un universo girando como un todo, en relación a ¿qué sistema referencial?.

A este respecto, Dirac sugirió una consecuencia muy interesante del descubrimiento de la radiación cósmica de fondo, interpretada como una radiación residual de la gran explosión que dio origen al universo según el modelo estándar. Por las características de anisotropía de la radiación de fondo, se podría deducir nuestro estado de movimiento con respecto al punto del espacio-tiempo en que se originó la explosión. Por extrapolación se podría entonces determinar un sistema de referencia en reposo con respecto al átomo original, lo cual pondría este sistema en una posición de privilegio, que se asume prohibido por la relatividad especial.

Sin embargo Wigner, al respecto, hizo notar que la relatividad especial sólo dice que las leyes de la naturaleza son las mismas en todos los sistemas que se mueven con velocidad uniforme unos respecto de los otros y ligados entre sí a través de las transformaciones de Lorentz, pero no afirma nada con respecto a las condiciones iniciales. De allí que el descubrimiento de un sistema de referencia en reposo con respecto al gran átomo original no contradice a la relatividad especial.

\section{La mecánica relacional de A. K. T. Assis}

En su libro Mecánica Relacional 1998[1], Andre Koch Torres de Assis desarrolla una teoría donde se plantea la implementación cuantitativa del principio de Mach por un camino alternativo a la teoría de la relatividad general de Einstein. 
La esencia de la mecánica relacional es el postulado del equilibrio dinámico que dice:

La suma de todas las fuerzas de cualquier naturaleza (gravitacional, eléctrica, magnética, elástica, nucleares, y demás) actuando sobre cualquier cuerpo es siempre nula en todos los sistemas de referencia.

Desde un punto de vista axiomático, los postulados de esta nueva teoría son:

1. Las fuerzas satisfacen el principio de acción y reacción o tercera ley de Newton

2. La sumatoria de todas las fuerzas actuando sobre un cuerpo, es nula en todos los sistemas de referencia.

Para la implementación del primer postulado, deberá asumirse como interacciones electromagnéticas y gravitacionales las leyes propuestas por Wilhelm Weber [3], esta es una fuerza central como la de Newton, que satisface el principio de acción y reacción y se dirige a lo largo de la línea que une los cuerpos. Además de esto es completamente relacional, o sea, depende solo de la distancia entre los cuerpos $r$, y de sus derivadas temporales $(d r)$ $d t$ y $d^{2} r / d t^{2}$ ), por esto tiene el mismo valor numérico en todos los sistemas de referencia.

En esta teoría para derivar la ecuación de movimiento de una partícula de prueba es necesario calcular su interacción con el universo distante, es en esta derivación donde se puede ver la mayor diferencia con las mecánicas de Einstein y Newton, se supone un sistema de referencia donde el cascarón vacío de masa $M$ y radio $R$ este en reposo, si se estudia una partícula de prueba de masa $m$ siendo acelerada en el interior del cascarón por otros cuerpos, entonces la fuerza gravitacional ejercida por el cascarón sobre la partícula de acuerdo con la fuerza de Weber, estará dada por kma.

En esta expresión $k$ es una constante que depende del radio y de la masa del cascarón y $a$ es la aceleración de la partícula en relación al cascarón, tanto en la mecánica de Einstein como en la de Newton esta fuerza debe ser nula.

Al estudiar la caída libre de un cuerpo en la mecánica relacional se debe tener en cuenta su interacción con todo el universo conocido, al hacer esto se obtiene una expresión análoga al asegunda ley de movimiento de Newton, con algunas ventajas [2].

En primer lugar, se deriva el principio de equivalencia (piedra angular de la relatividad general) o sea la proporcionalidad (equivalencia) entre la masa inercial y la gravitacional.

Se deriva de esta ley también que el mejor marco de referencia inercial es el de las galaxias distantes, al integrarse de forma análoga la energía potencial de Weber, se obtiene también la energía cinética como una energía gravitacional de cualquier cuerpo interactuando con el universo distante, también se obtiene una relación entre la constante de Hubble y la constante de gravitación universal $G$, y la densidad de materia del universo, esta relación es la misma deducida por Dirac desde los años treinta, sin que se hubiese dado una explicación satisfactoria para ella, finalmente también aparecen de esta ley las fuerzas centrífuga y de Coriolis en todos los sistemas de referencia en los que el Universo distante está girando.

Se muestra también en este libro [1] detalladamente, que todos los cuatro puntos, planteados por Einstein son incorporados por la mecánica relacional, y en particular la concavidad de la superficie del agua en el balde de Newton se debe a la rotación relativa entre el agua y los cuerpos celestes distantes. La concavidad es vista en esta teoría como un efecto de interacción gravitacional entre el agua y el universo cuando hay una rotación 
relativa entre ellos, se muestra matemáticamente que aunque el balde con agua este quieto con relación a la tierra y girasen las estrellas y las galaxias distantes alrededor del eje del balde, el agua subirá por las paredes del balde tomando una forma parabólica, por tanto según el autor por primera vez se implementa matemáticamente el principio de Mach.

Assis propone, a la vez, el llamado principio de proporciones físicas, "los fenómenos físicos solo pueden depender de la razón de las magnitudes físicas, mas no del valor absoluto de cualquiera de ellas" respecto de la experiencia del balde de Newton, cómo las dos situaciones son cinematicamente equivalentes, o sea con el mismo movimiento relativo entre el agua y las estrellas, no es posible diferenciarlas dinámicamente, en términos más amplios según la mecánica relacional las visiones ptolemaica y Copernicana son equivalentes no solo desde la perspectiva visual, sino también dinámico (la Tierra se achata desde los dos puntos de vista, el péndulo de Foucault precede de la misma forma en estos dos paradigmas).

Con el principio de equilibrio dinámico se da cumplimiento a la afirmación de Mach cuando dice: "Todas las masas y todas las velocidades y por consiguiente todas las fuerzas, son relativas".

\section{Conclusiones críticas}

Aunque la mecánica relacional vincula matemáticamente el principio de Mach, lo hace sacrificando importantes principios de la física.

Una de las contradicciones de la mecánica relacional está en que se fundamenta en la generalización de la fuerza de Weber para la gravedad, esta fuerza es una fuerza instantánea y de acción a distancia, invariante solo bajo las transformaciones de Galileo; dado que ninguna fuerza de acción a distancia puede ser invariante ante las transformaciones de Lorentz, (la noción de simultaneidad es inherente al observador, según la teoría especial de la relatividad). La no invariancia ante las transformaciones de Lorentz, vulnera el principio de simetría de las leyes de la física altamente estimado como atributo de una teoría física contemporánea.

La electrodinámica de Weber, basada en una fuerza de acción a distancia e instantánea, también contradice datos experimentales bien conocidos sobre la aceleración de cargas eléctricas, como la aceleración de los electrones en una antena bipolar donde no se produce una interacción instantánea.

La teoría electromagnética de Maxwell, basada en numerosas experiencias, predice y explica satisfactoriamente la existencia de ondas electromagnéticas que se propagan con la velocidad de la luz en el vacío, por tanto desde el punto de vista experimental es mucho más satisfactoria esta electrodinámica que la de Weber, además de ser invariante bajo las transformaciones de Lorentz (exigencia del principio de simetría de las leyes de la física).

También ha sido demostrado que la fuerza electrodinámica de Weber predice una dirección incorrecta para la radiación de una carga acelerada y que no puede explicar la radiación sincrotrónica, confirmada ampliamente en los aceleradores de partículas, dado que la fuerza de Weber solo tiene en cuenta aceleraciones radiales. 
Si se propone un desarrollo de la mecánica desde el punto de vista planteado por la mecánica relacional de Assis, es necesario hacer caso omiso de hechos experimentales suficientemente bien establecidos, y nos regresa a un escenario de discusión en torno a la teoría del éter cuando se buscaba, a través de este, explicar la propagación de las ondas electromagnéticas.

El paradigma que se enseña en los cursos de mecánica y electrodinámica, esta basado tanto en la mecánica newtoniana, en la electrodinámica de Maxwell y en la teoría especial de la relatividad de Einstein, la inclusión de una nueva visión en un curso de mecánica a nivel universitario, implicaría grandes cambios en su metódica de enseñanza, facilitaría eso sí, el enfoque crítico de la enseñanza de las ciencias, y en general tendría consecuencias pedagógicas por explorar, pero, ¿cuales implicaciones conceptuales podría tener? Es una pregunta que no puede pasar desapercibida, máxime cuando la mecánica relacional de Assis, no describe satisfactoriamente los fenómenos naturales observados, ni tampoco predice nuevos efectos y fenómenos, lo que si han hecho satisfactoriamente las teorías especial y general de la relatividad.

Una falencia importante adicional de la nueva mecánica, está en que no desarrolla ni asume el concepto de sistema de referencia, concepto del que hace caso omiso desde sus fundamentos, por tanto sus implicaciones tampoco aparecen suficientemente claras, como sí sucede en la relatividad general, en la cual, con posterioridad a su formulación ha sido desarrollado este concepto desde el punto de vista algebraico, geométrico y geometrofísico [7].

Sin embargo, una mecánica relacional que vincula el principio de Mach, que es coherente en alto grado a pesar de contradecir importantes datos experimentales, podría mejorar la comprensión de problemas cosmológicos importantes como la naturaleza de la materia oscura, la geometría del universo, la naturaleza del espacio y el tiempo, siempre será una buena razón para la discusión científica, lógica y epistemológica y digna de resaltar como una nueva conquista del pensamiento científico.

\section{Referencias bibliográficas}

1. Assis, A.K.T.(1998) Mecánica Relacional. Centro de Lógica, Epistemología e historia de la Ciencia-UNICAMP 1998

2. Assis, A.K.T. (1989) “On Mach`s principle” Found. Phys. Lett. 2 301-318

3. Assis, A.K.T (1995), Electrodinámica de Weber. Teoría aplicaciones y ejercicios. Campinas: Ed da Unicamp.

4. Mach E.(1949) Desarrollo Histórico-Crítico de la mecánica, Espasa

5. Einstein, A.(1922)El significado de la Relatividad. Traducción de miguel paredes larrueca 1961, Planeta Agostini.

6. Newton I. (1687) Principios Matemáticos, traducción Antonio Escotado, Grandes Obras del Pensamiento, Barcelona, 1.994

7. Vladimirov Y.S.(2005) Geometrofísica, Editorial BIMON Moscú (en ruso)

8. Mashhoon B, Dorio L and Lichtenegger, Phys, Lett. A 292, 49 (2201) 
9. Schlick M, Zeitschrift für Philosophie und Philosophische kritik 159, 164 (1915)

Recibido 16/10/07. Aprobado 28/11/07 